\title{
Changes in smoke alarm coverage following two fire department home visiting programs: what predicts success?
}

Andrea C Gielen*, Elise C Perry, Wendy C Shields, Eileen McDonald, Shannon Frattaroli and Vanya Jones

\begin{abstract}
Background: Door-to-door canvassing and installation of smoke alarms have been found to be effective at increasing the number of homes protected. This analysis reports on how smoke alarm coverage changes six months after a home visiting program in a large urban sample, and how this change varies by characteristics of the residents and characteristics of the services delivered during the home visit.

Methods: Fire department Standard and Enhanced home visiting programs were compared. During the home visit, fire fighters installed lithium battery smoke alarms. Residents in the Enhanced program received tailored education about fire safety. Six months after the home visit, participating residences were visited to complete a follow-up survey and to have the installed alarms checked.

Results: $81 \%$ of the 672 homes that had a working smoke alarm on every level of the home at the end of the home visit remained safe at follow-up, and $87 \%$ of the residents found the home visit was very useful, and these rates did not differ between the Enhanced and Standard programs. The degree to which firefighters delivered their services varied, although households in which the resident's engagement with the fire department team was rated as excellent were 3.96 times as likely to be safe at follow-up compared to those with poor or fair resident engagement $(\mathrm{p}=0.03)$.

Conclusions: There is a need to better understand how to maximize the time spent with residents during smoke alarm home visiting programs. This study helps with the development of methods needed for implementing and evaluating such programs in real-world settings.
\end{abstract}

Keywords: Injury prevention; Smoke alarms; Fires and burns; Home visits; Community health workers; Community intervention

\section{Background}

Residential fires present a threat to the public's health and safety. In 2012, there were approximately 365,000 fires occurring in home structures resulting in 2,380 civilian deaths and 12,875 civilian injuries (Karter 2012). Low-income neighborhoods are at substantially higher risk, in part due to the presence of older and vacant housing that increases fire risk, and high proportions of immigrant populations with limited ability to read and speak English that decreases potential impact of

\footnotetext{
* Correspondence: agielen@jhsph.edu

Johns Hopkins Bloomberg School of Public Health, Johns Hopkins Center for Injury Research and Policy, 624 N. Broadway, Baltimore, MD 21205, USA
}

communication about preventive measures (Istre et al. 2001; Shai 2006; Schachterle et al. 2012).

Working smoke alarms reduce the risk of death in the event of a house fire by $50 \%$ (Istre et al. 2001). Having a working smoke alarm with a long-lasting lithium battery on every level of the home is the recommended best practice according to the Centers for Disease Control and Prevention (Ballesteros et al. 2005). In an observational survey of homes in Baltimore City, 97\% had at least one working smoke alarm, but less than half had one on every level (Stone et al. 2007). An estimated 20$50 \%$ of smoke alarms in homes are non-functional, and many residents do not know if their smoke alarms are working (Sidman et al. 2011; Stepnitz et al. 2012). The 
percentage of homes with functioning smoke alarms ranges from 34\%-93\% among high-risk communities (Liu et al. 2011).

Door-to-door canvassing and in-home installation of smoke alarms have been found to be the most effective method for increasing the number of homes protected. (Ta et al. 2006) In a meta-analysis, the most intensive smoke alarm programs, those with a combination of education, low cost or free equipment and direct installations, and those programs that installed lithium battery alarms reported the highest rates of coverage (Cooper et al. 2012). The prevalence of working smoke alarms after installation has been reported between $79 \%$ - 92\% at 12 months and $64 \%-82 \%$ at 3-4 years (Ta et al. 2006; Yang et al. 2008; Peek-Asa et al. 2010).

Fire departments and other community organizations that want to increase the prevalence of working smoke alarms in their neighborhoods could benefit from knowing more about how such programs can be most effectively implemented. In this study, the following questions are addressed: 1) How does smoke alarm coverage change six months after a home visiting program in a large urban sample? 2) How does this change in smoke alarm coverage vary by characteristics of the residents and characteristics of the services delivered during the home visit?

\section{Methods}

\section{Study design}

The Johns Hopkins Home Safety Project was a partnership with the Johns Hopkins Center for Injury Research and Policy (JHCIRP), Baltimore City Fire Department (BCFD), Environmental Justice Partnership, and the Urban Health Institute. As previously described, 12 census tracts were selected in East Baltimore -6 were randomly assigned to receive the Standard BCFD home visiting program and 6 were randomly assigned to receive an Enhanced home visiting program (described below) (Gielen et al. 2013). From April 2010 to April 2011, a total of 171 canvassing events occurred (82 in the Standard and 89 in the Enhanced areas) during which the firefighters went door-to-door to deliver the home visiting programs. The firefighters came from 21 different fire companies, and were assigned by the BCFD based on the jurisdiction of their fire station and the area to be canvassed. Over the course of the study, the firefighters could have visited homes receiving either the Enhanced or the Standard program, but the distinction was not pointed out to them because the protocol for the firefighters was the same in both. Residents who accepted the home visit and completed both a baseline and six-month follow up survey are the subjects of this analysis, making this a quasi-experimental study design.

\section{Standard home visiting program}

Based on formative work done by the partnership at the outset of the project (Frattaroli et al. 2012), the BCFD created a specific home visiting protocol that was adopted throughout the Department. Training in the new protocol was provided to all firefighters either through in-person training provided by the Fire Marshal's Office, or for those firefighters who could not attend the training, the materials were provided for them to review on their own. The protocol included having teams of four BCFD firefighters going door-to-door in their assigned neighborhoods and offering free alarms, which they installed on every level of the home; they also tested CO levels in the home. To provide information and encouragement to maintain working smoke alarms, the firefighters educated the resident about the smoke alarms' long-lasting batteries, their hush feature, how to use alarm, fire prevention (cooking, electrical, heating issues), fire escape planning, and $\mathrm{CO}$ safety. Residents received the smoke alarm instruction manual, and a home safety checklist. The smoke alarms were obtained from the Maryland Department of Health and Mental Hygiene.

\section{Enhanced home visiting program}

Firefighters followed the same protocols for installation and resident education as in the Standard program, with the following additions: 1) community health workers (CHWs) went door-to-door one week in advance of the fire department's visit and on the day of the visit to inform residents about the free services, answer residents' questions about services, and encourage residents to participate; 2) a safety educator visited the home with the firefighters to provide tailored home safety education on hot water temperatures and $\mathrm{CO}$ poisoning; and 3) a mobile safety center with interactive educational exhibits and low cost safety products parked in the neighborhood on the day of the fire department's visit, and residents were invited to visit it (Bulzacchelli et al. 2009; Gielen et al. 2009).

\section{Hypotheses and rationale}

For this analysis, we hypothesized that smoke alarm coverage at six-month follow-up would be higher among residents in the Enhanced home visiting program compared to those in the Standard home visiting program. The difference between these two programs is that those in the Enhanced program received additional education from a safety educator who accompanied the firefighters, and we assumed that would increase the resident's motivation to maintain their alarms in good working order. Second, we hypothesized that smoke alarm coverage at the six-month follow-up would be higher when there was greater compliance with the home visit protocol by the firefighters, and when the residents responded more 
favorably to the visit. This hypothesis assumes that the resident's motivation to maintain their smoke alarms would be greater when they responded more positively to having the firefighters in their homes, and the better the firefighters were at completing all the installation and education tasks. We had no hypothesis about the relationship between firefighter fidelity to implementing their tasks and resident response because the resident did not know what the firefighter protocols involved, although we do examine those potential relationships.

\section{Data collection}

The Johns Hopkins Institutional Review Board approved this study. Data collection took place inside residents' homes at the time of the home visit (baseline) and six months later (follow-up). Once permission to enter the home was given for the baseline home visit, the firefighters installed the smoke alarms while trained data collectors recorded their activities (number and location of alarms, fidelity to protocol, etc.). When the firefighters finished installing the alarms, the data collectors asked the resident to complete a brief survey about their home visit experience and their home safety knowledge (the baseline survey). If residents declined this baseline survey, they were not offered a follow-up visit. If residents completed the baseline survey, they were informed about the six-month follow-up and asked if they would be willing to participate. Over the course of the study 49 data collectors were employed, all of whom were trained in the data collection protocols through an in-office training program followed by two in-the-field sessions in which they shadowed a veteran data collector. They were assigned to the home visits based on their availability, and could collect data in both the Standard and Enhanced groups, where the fire department protocols were identical.

In the Standard program, the visit ended after residents completed the baseline survey. In the Enhanced program, responses to the baseline survey were used by the safety educator to provide specific educational messages tailored to the responses (McDonald et al. 2013). The extra education on carbon monoxide and hot water scald burns provided by the safety educator was the main difference in the home visit experience between the Enhanced and Standard programs. The mobile safety center was not able to attend all of the canvassing events and there was insufficient uptake of it when it was present, so no further data from that component of the program is considered here.

Six months after the home visit, each participating residence was visited to complete the follow-up survey and to have all the installed alarms checked. Homes were visited up to ten times on nonconsecutive days during daylight hours until the resident completed or refused the follow- up or they were deemed ineligible. Respondents were ineligible if the original respondent had moved, the home was vacant, or the respondent was impaired and unable to complete the follow up visit. The remainder who were lost to follow-up were those whose survey windows expired (ten attempts by data collectors to reach the participant and complete the survey).

\section{Measures \\ Sociodemographic characteristics of residents}

Sociodemographic information was collected during the follow-up interview. Residents self-reported information about household size, composition, and income; respondent age, education, employment, and ethnicity.

\section{Characteristics of the home visit}

Implementation fidelity (smoke alarm, education) During the home visit, data collectors observed fire department activities and recorded fidelity to the fire department's protocol using a process evaluation checklist. The checklist covered both smoke alarm installation and safety education expectations for fire personnel.

The smoke alarm section had five tasks the fire department personnel were to complete: 1) tell resident about the long-lasting battery; 2) describe the alarm's hush feature to the resident; 3 ) demonstrate how to use alarm; 4) give the instruction manual to the resident; and 5) install the alarms using screws. The data collector recorded a yes/no for each item, which resulted in a score of 0-5 on the smoke alarm implementation fidelity score.

The safety education section included six tasks: 1) discuss $\mathrm{CO}$ safety; 2) discuss fire escape planning; 3) discuss cooking safety; 4) discuss electrical safety; 5) discuss heating safety; and 6) distribute a home safety checklist that reinforced this information. The data collector recorded a yes/no for each item, which resulted in a score of 0-6 on the education implementation fidelity score.

Observer reported ratings at the home visit (Fire Department, Resident Engagement) At the end of the home visit, data collectors provided their subjective assessment of the home visit by rating the overall quality of the visit and the residents' engagement with the fire department personnel (poor, fair, good, very good, or excellent). Based on the distribution of responses, the categories were recoded for analysis with "poor" and "fair" combined.

Resident reported ratings at follow-up During the follow up, interviewers asked residents to rate how useful the home visit was to them (not at all, just a little, somewhat or very useful). Based on the distribution of responses, the categories were recoded for analysis with "not at all" and "just a little" combined. 


\section{Observed safety behaviors}

During the original home visit, data collectors recorded the number and location of all smoke alarms installed by the BCFD. During the follow up visit, data collectors recorded the location of each of the installed alarms and tested them by pushing the test button. A summary variable was created to indicate whether there was a working smoke alarm (of any type) on every level of the home at baseline and at follow-up. At both time points, a home was considered to have "all levels safe" if there was a working smoke alarm (of any type) on every level of the home. At baseline homes were coded as "not having all levels safe" if the firefighter did not install an alarm on every level (most often because the resident did not allow access to a level); and, at follow up, if the installed alarms were either missing or non-functioning.

\section{Statistical analysis}

Demographic characteristics of the study sample were tabulated and compared between the enhanced and standard study conditions. A paired t-test was used to compare changes in smoke alarm coverage in the two study groups. The two study groups were compared on receipt of the home visit components and resident reactions, using rank sum statistics. Rank sum tests were used because the outcome has ordinal response categories. Finally, multiple logistic regression was used to identify correlates of having working smoke alarms on all levels at the six-month follow-up, including covariates on which the two study groups differed, smoke alarm status at baseline, and variables that were significant in bivariate analyses (data not shown). Number of people in the home was excluded from the model because of its correlation with having children in the home.

\section{Results \\ Sample}

As shown in Figure 1, 983 residents in the standard program and 1214 in the enhanced program areas participated in the original home visits. Of these, 680 (69.18\%) in the standard program and $709(58.40 \%)$ in the enhanced program agreed to complete the baseline survey $(p<0.01)$, which made them eligible to recruit for the sixmonth follow-up. Of those eligible for the follow-up, 633 (93.08\%) and $629(88.72 \%)$ accepted in the standard and enhanced areas respectively $(\mathrm{p}<0.01)$. Between January 2011 and December 2011, 754 follow-up interviews were completed (381 in the enhanced and 373 in the standard communities).

There was no difference in the completion rates across groups for the follow-up survey: $373(58.92 \%)$ in the standard area and $381(60.57 \%)$ in the enhanced $(\mathrm{p}=0.55)$ completed the follow-up. Ninety-six (15.16\%) people in

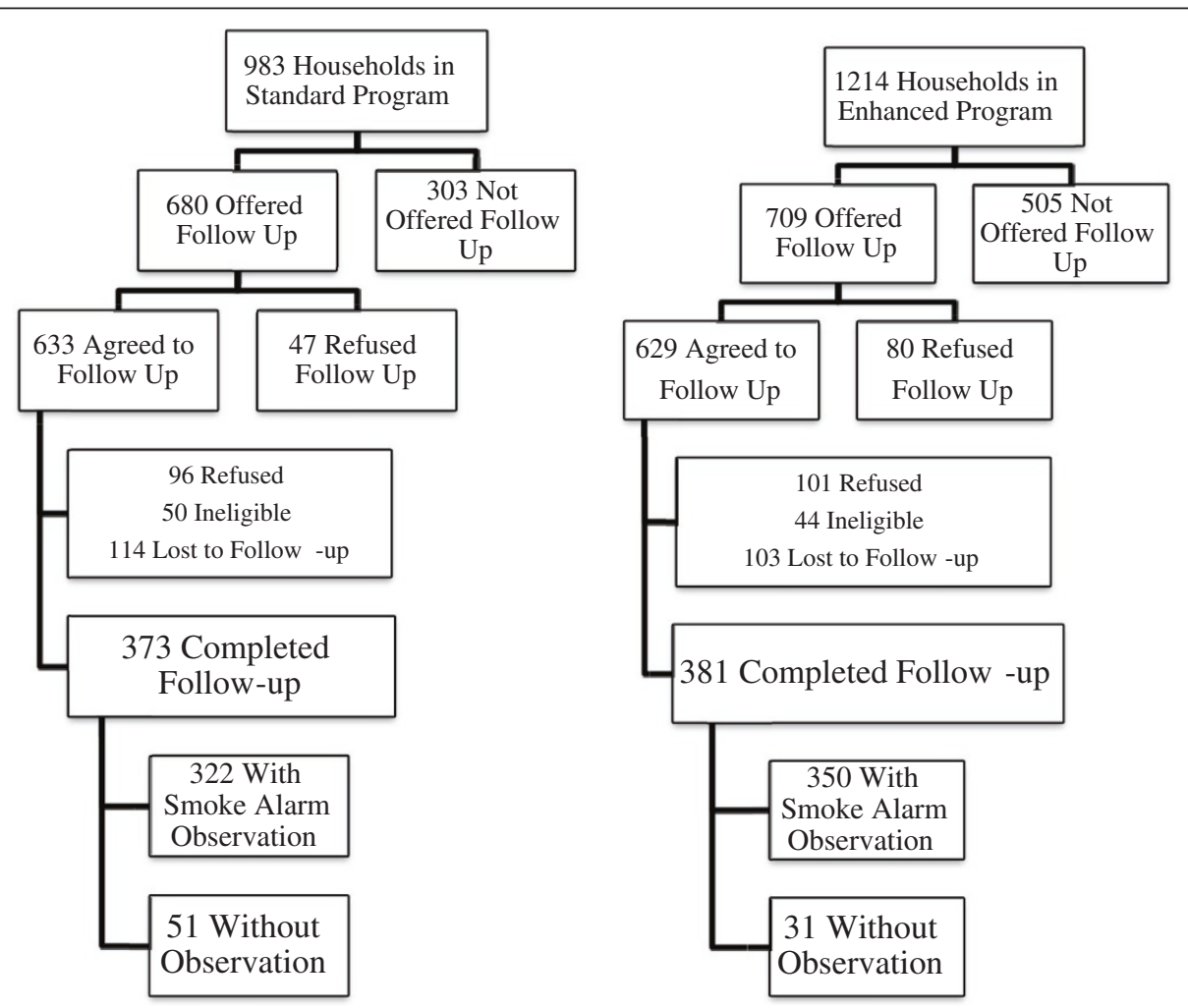

Figure 1 Study households participation flow chart. 
Table 1 Sociodemographic characteristics of follow-up participants: comparing the standard and enhanced programs

\begin{tabular}{|c|c|c|c|c|c|}
\hline & & $\begin{array}{l}\text { Standard } \\
\mathrm{N}=341(\%)\end{array}$ & $\begin{array}{l}\text { Enhanced } \\
\mathrm{N}=367(\%)\end{array}$ & $\begin{array}{l}\text { Total } \\
\mathrm{N}=708^{1}(\%)\end{array}$ & Chi-square \\
\hline \multirow[t]{2}{*}{ Gender } & Male & $82(24.05)$ & $110(29.97)$ & $192(27.12)$ & $3.14(p=0.08)$ \\
\hline & Female & $259(75.95)$ & $257(70.03)$ & $516(72.88)$ & \\
\hline \multirow[t]{5}{*}{ Age } & 18 to 24 & $13(3.82)$ & $14(3.84)$ & $27(3.83)$ & $12.89(p=0.01)$ \\
\hline & 25 to 34 & $85(25.00)$ & $60(16.44)$ & $145(20.57)$ & \\
\hline & 35 to 44 & $58(17.06)$ & $74(20.27)$ & $132(18.72)$ & \\
\hline & 45 to 55 & $80(23.53)$ & $72(19.73)$ & $152(21.56)$ & \\
\hline & 55 and above & $104(30.59)$ & $145(39.73)$ & $249(35.32)$ & \\
\hline \multirow[t]{2}{*}{ Household Role } & Head of Household & $289(84.75)$ & $302(82.74)$ & $591(83.71)$ & $0.52(p=0.50)$ \\
\hline & Other & $52(15.25)$ & $63(17.26)$ & $115(16.29)$ & \\
\hline \multirow[t]{4}{*}{ Education } & $<$ high school diploma & 59 (17.35) & $76(20.82)$ & $135(19.15)$ & $3.80(p=0.28)$ \\
\hline & HS diploma/GED & $128(37.65)$ & $145(39.73)$ & $273(38.72)$ & \\
\hline & Some college & $67(19.71)$ & $54(14.79)$ & $121(17.16)$ & \\
\hline & Completed college & $86(25.29)$ & $90(24.66)$ & $176(24.96)$ & \\
\hline \multirow[t]{2}{*}{ Household income below the poverty line? } & Yes & $75(26.69)$ & $83(27.21)$ & $158(26.96)$ & $0.02(p=0.89)$ \\
\hline & No & $206(73.31)$ & $222(72.79)$ & $428(73.04)$ & \\
\hline \multirow[t]{2}{*}{ Homeowner Status } & Rent & $144(42.73)$ & $148(40.66)$ & $292(41.65)$ & $0.31(p=0.58)$ \\
\hline & Own or pay mortgage & $193(57.27)$ & $216(59.34)$ & $409(58.35)$ & \\
\hline \multirow[t]{2}{*}{ Children in home $(<18 \mathrm{y})$} & Yes & $180(52.79)$ & $137(37.33)$ & $317(44.77)$ & $17.07(p<0.01)$ \\
\hline & No & $161(47.21)$ & $230(62.67)$ & $391(55.23)$ & \\
\hline \multirow[t]{4}{*}{ Number of people in the home } & 1 person & $34(10.00)$ & $77(21.10)$ & $111(15.74)$ & $18.01(p<0.01)$ \\
\hline & 2-3 people & $169(49.71)$ & $174(47.67)$ & $343(48.65)$ & \\
\hline & 4-6 people & $119(35.00)$ & $98(26.85)$ & $217(30.78)$ & \\
\hline & 7 or more people & $18(5.29)$ & $16(4.38)$ & $34(4.82)$ & \\
\hline
\end{tabular}

${ }^{1} 46$ residents did not respond to survey questions; some variables do not add up to 708 due to missing item responses.

the standard area and $101(16.06 \%)$ in the enhanced area refused the follow up survey; $50(7.90 \%)$ in the standard area and $44.7 .00 \%)$ in the enhanced area were ineligible.

Follow-up participants in the Standard program were more likely to be older, and to have children and a larger number of people living in the home relative to households in the Enhanced program (Table 1).

\section{Smoke alarm changes from baseline home visit to} 6-month follow-up

In the Standard program 288 (89.44\%) homes had all levels safe after the home visit (baseline) compared to
324 (92.57\%) homes in the Enhanced program. (Table 2) In the Standard program homes at follow-up, 235 (72.98\%) had all levels safe compared to 274 (78.28\%) in the Enhanced program homes.

Table 2 examines the proportion of homes with all levels safe at follow-up as a function of their status at baseline. For the entire sample, $80.88 \%$ of homes that were safe at the end of the home visit remained safe at follow-up. In the Standard program homes, $78.47 \%$ that were safe at baseline were safe at the six month followup, compared to $83.02 \%$ of homes in the Enhanced program. Of the homes that did not have all levels safe at

Table 2 Smoke alarm coverage at baseline and follow-up: comparing the standard and enhanced programs ${ }^{1}$

\begin{tabular}{|c|c|c|c|c|c|c|c|c|c|c|}
\hline \multirow[t]{2}{*}{$\begin{array}{l}\text { All levels } \\
\text { safe? }\end{array}$} & & \multicolumn{3}{|c|}{$\begin{array}{l}\text { Standard program } \\
\text { Follow up }(\mathrm{N}=322)\end{array}$} & \multicolumn{3}{|c|}{$\begin{array}{l}\text { Enhanced program } \\
\text { Follow up }(\mathrm{N}=350)\end{array}$} & \multicolumn{3}{|c|}{$\begin{array}{l}\text { Total sample } \\
\text { Follow up }(\mathrm{N}=672)^{3}\end{array}$} \\
\hline & & Yes & No & Total & Yes & No & Total & Yes & No & Total \\
\hline \multirow[t]{2}{*}{ Baseline } & Yes & $226(78.47 \%)$ & $62(21.53 \%)$ & $288(100 \%)$ & $269(83.02 \%)$ & 55 (16.97\%) & $324(100 \%)$ & $495(80.88 \%)$ & $117(19.12 \%)$ & $612(100 \%)$ \\
\hline & No & 9 (26.47\%) & $25(73.53 \%)$ & 34 (100\%) & $5(19.23 \%)$ & $21(80.77 \%)$ & $26(100 \%)$ & $14(20.33 \%)$ & 46 (76.67\%) & $60(100 \%)$ \\
\hline Total & & 235 (72.98\%) & 87 (27.02\%) & 322 (100\%) & $274(78.28 \%)$ & $76(21.71 \%)$ & $350(100 \%)$ & 509 (75.74\%) & 163 (24.26\%) & $672(100 \%)$ \\
\hline
\end{tabular}

${ }^{1}$ Paired t-test for changes from baseline to follow-up between Standard versus Enhanced: $t=0.68(p=0.50)$.

${ }^{2}$ All Levels Safe was defined as working long life battery operated or hard-wired alarms on all levels of the home.

${ }^{3}$ Smoke alarm status at follow up was unavailable for 51 households in the standard and 31 households in the enhanced area. 
Table 3 Ratings in-home intervention components by observers and residents: comparing standard and enhanced study programs

\begin{tabular}{|c|c|c|c|c|c|}
\hline \multicolumn{2}{|l|}{ Observer ratings at baseline home visit } & $\begin{array}{l}\text { Standard } \\
\mathrm{N}=373 \\
\text { Mean \% (SD) }\end{array}$ & $\begin{array}{l}\text { Enhanced } \\
\mathrm{N}=381 \\
\text { Mean\% (SD) }\end{array}$ & $\begin{array}{l}\text { Total } \\
\mathrm{N}=754^{1} \\
\text { Mean\% (SD) }\end{array}$ & Test statistic \\
\hline \multirow{4}{*}{ Fire Department Implementation } & & & & & \\
\hline & Smoke Alarm Fidelity² & $57.97(22.26)$ & $60.84(22.25)$ & $59.51(23.93)$ & Rank Sum $(p=0.16)$ \\
\hline & Education Fidelity $^{3}$ & $19.91(20.02)$ & 23.72(23.14) & $21.85(21.73)$ & Rank Sum ( $p=0.05)$ \\
\hline & & $\mathrm{N}(\%)$ & $\mathrm{N}(\%)$ & $\mathrm{N}(\%)$ & \\
\hline \multirow{4}{*}{$\begin{array}{l}\text { How would you rate the FD's delivery } \\
\text { of services? }\end{array}$} & Poor or Fair & $108(29.59)$ & $93(24.67)$ & $201(27.09)$ & Rank Sum $(p=<0.01)$ \\
\hline & Good & $151(41.37)$ & $137(36.34)$ & $288(38.81)$ & \\
\hline & Very Good & $86(23.56)$ & $100(26.53)$ & $186(25.07)$ & \\
\hline & Excellent & $20(5.48)$ & $47(12.47)$ & $67(9.03)$ & \\
\hline \multirow{4}{*}{$\begin{array}{l}\text { How would you rate the resident's } \\
\text { engagement with the FD team? }\end{array}$} & Poor or Fair & $46(12.57)$ & $21(5.57)$ & 67 (9.02) & Rank Sum $(p=0.10)$ \\
\hline & Good & $157(42.90)$ & $182(48.28)$ & $339(45.62)$ & \\
\hline & Very Good & $128(34.97)$ & 127 (33.69) & $255(34.32)$ & \\
\hline & Excellent & $35(9.56)$ & $47(12.47)$ & $82(11.04)$ & \\
\hline Resident ratings at follow-up & & Standard N = 311 & Enhanced $\mathrm{N}=341$ & Total $\mathrm{N}=652^{4}$ & \\
\hline \multirow{3}{*}{$\begin{array}{l}\text { Thinking back to the home visit, } \\
\text { how useful would you say it } \\
\text { was for you? }\end{array}$} & Very useful & $266(86.08)$ & $295(87.28)$ & $561(86.71)$ & Rank Sum $(p=0.61)$ \\
\hline & Somewhat useful & $33(10.68)$ & $37(10.95)$ & $70(10.82)$ & \\
\hline & Just a little or not at all & $10(3.24)$ & $6(1.78)$ & $16(2.47)$ & \\
\hline
\end{tabular}

${ }^{1}$ Some variables do not add up to 754 due to missing item responses.

${ }^{2}$ Based on 5 components (tell resident about 10 year battery, show hush feature, show how to use alarm, give instruction manual to resident, screw in alarms).

${ }^{3}$ Based on 6 components (distributed checklist, discuss CO safety, discuss escape plan, discuss cooking safety, discuss electrical safety, discuss heating safety).

${ }^{4}$ Of the 708 residents completing the follow up survey, 652 recalled the fire department home visit and answered this question.

Table 4 Smoke alarm coverage six months after home installation program: multiple logistic regression model of all levels of the home safe at follow-up, $N=626$

\begin{tabular}{|c|c|c|c|}
\hline & & $\begin{array}{l}\text { All Levels Safe at } \\
\text { Follow-up }\end{array}$ & $\overline{p \text {-value }}$ \\
\hline \multirow[t]{2}{*}{ Program } & Standard & Reference & \\
\hline & Enhanced & 1.08 & 0.72 \\
\hline \multirow[t]{2}{*}{ Smoke Alarm Safety at Baseline } & Not Safe & Reference & \\
\hline & Safe & 17.06 & $<0.01$ \\
\hline \multirow[t]{5}{*}{ Age of Respondent } & 18 to 24 & Reference & \\
\hline & 25 to 34 & 1.87 & 0.50 \\
\hline & 35 to 44 & 2.93 & 0.19 \\
\hline & 45 to 54 & 2.31 & 0.73 \\
\hline & 55 and above & 3.61 & 0.01 \\
\hline \multirow[t]{2}{*}{ Household Role } & Head of Household & Reference & \\
\hline & Other & 0.63 & 0.09 \\
\hline \multirow[t]{2}{*}{ Children in home? } & Yes & Reference & \\
\hline & No & 1.07 & 0.77 \\
\hline \multirow{4}{*}{$\begin{array}{l}\text { How would you rate the resident's } \\
\text { engagement with the FD team? }\end{array}$} & Poor or Fair & Reference & \\
\hline & Good & 1.78 & 0.54 \\
\hline & Very Good & 2.22 & 0.57 \\
\hline & Excellent & 3.96 & 0.03 \\
\hline
\end{tabular}

\footnotetext{
${ }^{1}$ All levels safe defined as working long lasting lithium battery alarms or hard wired alarms on all levels of the home.
} 
the end of the home visit, $26.47 \%$ of those in the Standard and $19.23 \%$ of those in the Enhanced were safe at the six-month follow-up, which could occur, for example if the resident added a new alarm or replaced a battery where it was needed. The changes from baseline to follow up between the Enhanced and Standard programs were not statistically significantly different $(t=0.68, p=0.50)$.

\section{Predictors of smoke alarm coverage at 6-month follow-up}

Table 3 compares characteristics of program implementation between the Standard and Enhanced programs. There was no difference in the fire department's Smoke Alarm fidelity score, with an overall average of $59.51 \%$ of the tasks completed across all the home visits. For the Education fidelity score, $23.72 \%$ of the tasks were completed on average in the Enhanced program compared to $19.91 \%$ in the Standard program ( $\mathrm{p}=.05)$. Overall, the group receiving the Enhanced program had higher ratings on the delivery of services $(p<0.01)$, and for the resident's engagement, the difference approached statistical significance $(\mathrm{p}=0.10)$.

There was no difference between the Standard and Enhanced programs in the residents' rating of the usefulness of the program ( $\mathrm{p}=0.61$ ), with $86.71 \%$ rating it as very useful (Table 3). In a separate bivariate analysis, Smoke Alarm fidelity, but not Education fidelity, was associated with the resident's usefulness rating of the home visit $(\mathrm{p}=0.01)$. Participants reporting the home visit was "Very Useful" had the highest mean Smoke alarm fidelity $(58.36 \%)$, compared to those who responded the home visit was "Somewhat Useful" (48.64\%) and "Not at all useful" (40.00\%).

Of all of these program implementation variables, only the observer's rating of the resident's engagement with the firefighters was significantly related to the outcome of having all levels safe at follow-up in the bivariate analyses $(\mathrm{p}<0.01)$. The proportions that were safe vs. unsafe were $14.86 \%$ vs. $85.14 \%$ among those rated "Excellent"; $21.65 \%$ vs $78.35 \%$ among those rated "Very Good"; $25.74 \%$ vs $74.26 \%$ among those rated "Good"; and $28.81 \%$ vs $71.19 \%$ among those rated as "Fair or Poor".

In the multiple logistic regression model predicting "all levels safe" at follow-up (Table 4), study group was not significant. Regardless of study group, if the home was safe (i.e., had working alarms on all levels) at the end of the original home visit, it was significantly more likely to be safe at follow up $(\mathrm{OR}=17.06, \mathrm{p}<0.01)$ compared to homes that were unsafe at the end of the home visit. Survey respondents who were 55 and older were 3.61 times as likely to be in a home that was safe at follow up relative to 18 to 24 year olds $(\mathrm{p}=0.01)$. Controlling for age and the other variables in the model, households in which respondents' engagement with the fire department team was rated as excellent were 3.96 times as likely to have all levels safe compared to those households rated as poor or fair engagement with the program $(p=0.03)$. There was no interaction between age and resident engagement (data not shown).

\section{Discussion}

Our analysis did not support the hypothesis that smoke alarm coverage at the six-month follow-up would be higher in the enhanced program because they received additional education from a safety educator who accompanied the firefighters. Overall, a high proportion of homes that had working smoke alarms on all levels at the end of the home visit installation maintained these alarms (81\%). This may be partially explained by the fact that tamper resistant, long-lasting lithium battery alarms with a hush feature were installed, presumably making it less likely that the batteries would be removed or fail during the six-month interval (Jackson et al. 2010; Yang et al. 2008: Peek-Asa et al. 2010; Cooper et al. 2012).

Our results only partially supported the second hypothesis concerning compliance with the home visit protocol by the firefighters and resident response to the visit. Those residents who were rated as more engaged with the fire department team were significantly more likely to have working alarms on all levels at follow-up, even when controlling for standard vs. enhanced program, smoke alarm status at baseline, and multiple demographic variables. However, no relationship between the firefighters' fidelity to the home visit protocol and having working smoke alarms at follow-up was observed, which may be due to the overall low levels of fidelity to the smoke alarm and education protocols, and the minimal amount of effort needed on the part of the resident to maintain the alarms. Why rates of fidelity to the smoke alarm and education protocols was low warrants further exploration, as do our findings related to the lack of a relationship between other elements of program implementation, resident ratings of the visit usefulness and program outcomes. Further research is needed to better understand the "active ingredients" of the home visit that engage the resident as well as other home visit outcomes such as safety knowledge.

The selectivity of the sample is a limitation. In a previous analysis (Gielen, et al. 2013), we showed that our standard and enhanced neighborhoods were comparable to each other on key variables (e.g., income below poverty, education), but were poorer and less well educated than Baltimore City as a whole. The sample analyzed here was drawn from those residents who were home on the day of the canvassing, and who let the fire department into their homes. While the fire department provided smoke alarms to all residents who let them in, we could only collect evaluation data from those who agreed to complete our data collection at the home visit and at follow up. 
Comparing our final sample to Baltimore City as a whole found that they maybe slightly more disadvantaged on income below the poverty line ( $26.96 \%$ vs $20.00 \%)$, and less so on completing high school (80.85\% vs $76.90 \%)$ (Gielen et al. 2012).

Our recruitment strategy resulted in residents having multiple opportunities to decline participation. Most refusals occurred at the time of the home visit when residents were asked to complete the baseline survey (a prerequisite for being offered the follow-up), perhaps because there was already so much activity and several people in the home. The slightly longer home visit experience for those in the enhanced program may have also contributed to higher refusals for follow-up in that group. Residents could decline the follow-up both when it was offered after the baseline or when it was attempted, and some follow-up visits were not completed due to nonworking telephones, moving, and lack of response after multiple attempts. Thus, the sample is self-selected, and our rates of smoke alarm maintenance may not be representative of the larger population in the sampled census tracts or in the City overall.

Our follow-up duration of six months is consistent with some previous studies, but not as long as would be ideal. Nevertheless, our rate of $81 \%$ is within the previously reported ranges across multiple follow-up periods (Ta et al. 2006; Yang et al. 2008; Peek-Asa et al. 2010). This result, combined with the finding that the vast majority of residents $(86.71 \%)$ reported that the home visit was very useful bodes well for long-term impact of fire department home visiting programs on fire deaths and injuries. A remaining challenge is to better understand whether and to what extent the educational component of a home visit can make a difference in preventing fires and other home injuries in the first place.

\section{Conclusions}

There is now a robust literature supporting the benefits of comprehensive smoke alarm installation programs in many types of populations, including rural and urban areas (Cooper et al. 2012; Liu et al. 2011; Ta et al. 2006; Yang et al. 2008; Peek-Asa et al. 2010; Gielen et al. 2013). We found that the vast majority of residents rated the home visit as very useful, and our measure of resident engagement with the firefighters during the home visit was significantly associated with having the recommended number of working smoke alarms at follow-up. However, our observers noted considerable variability in the degree to which the firefighters delivered their services, and little is known about firefighters' perspectives and the barriers and facilitators they face in adding home visiting to their skill set and work load (Frattaroli et al. 2012). Thus, it is time to better understand how to maximize the time spent with residents, and this study helps with the development of methods needed for implementing and evaluating home visiting programs in realworld settings.

\section{Competing interest}

The authors declare that they have no competing interest.

\section{Authors' contributions}

ACG served as principal investigator on the study and oversaw all aspects of the intervention delivery and evaluation; ECP was the project director and data analyst; WCS was the research manager and responsible of oversight of the data collection and management; EM was co-investigator with primary responsibility for the educational components of the in-home intervention; SF was co-investigator with primary responsibility for working with the fire department; VJ was co-investigator with primary responsibility for the community health worker component. All authors contributed to writing, reviewing, and approving the final manuscript.

\section{Acknowledgements}

This work was supported by the Centers for Disease Control and Prevention, National Center for Injury Prevention and Control, Grant No. R18CE001339, and by the National Institutes of Health, National Institute of Child Health and Human Development, Grant No. RO1HD059216. The authors wish to acknowledge and thank all of the community partner organizations and individuals that contributed to the Johns Hopkins Home Safety Study.

Received: 5 August 2014 Accepted: 21 October 2014

Published online: 24 November 2014

\section{References}

Ballesteros M, Jackson M, Martin MW. Working toward the elimination of residential fire deaths: the Centers for Disease Control and Prevention's Smoke Alarm Installation and Fire Safety Education (SAIFE) program. J Burn Care Rehabil. 2005; 26(5):434-39.

Bulzacchelli MT, Gielen AC, Shields WC, McDonald EM, Frattaroli S. Parental safety-related knowledge and practices associated with visiting a mobile safety center in a low-income urban population. Fam Community Health. 2009; 32(2):147-58.

Cooper NJ, Kendrick D, Achana F, Dhiman P, He Z, Wynn P, Le Cozannet E, Saramago P, Sutton AJ. Network meta-analysis to evaluate the effectiveness of interventions to increase the uptake of smoke alarms. Epidemiol Rev. 2012; 34(1):32-45.

Frattaroli S, McDonald EM, Tran NT, Trump AR, O'Brocki RC 3rd, Gielen AC. Igniting interest in prevention: using firefighter focus groups to inform implementation and enhancement of an urban canvassing program. J Public Health Manag Pract. 2012; 18(4):382-89.

Gielen AC, McDonald E, Frattaroli S, McKenzie LB, Backes B, Glenshaw M, Shields W, Bulzacchelli MT. CARES (Children ARE Safe) Mobile Safety Center Partnership. If you build it, will they come? Using a mobile safety centre to disseminate safety information and products to low-income urban families. Inj Prev. 2009; 15(2):95-9.

Gielen AC, Shields W, Frattaroli S, McDonald EM, Jones V, Bishai D, O'Brocki R, Perry E, Bates-Hopkins B, Tracey P, Parsons S. Enhancing fire department home visiting programs: results of a community intervention trial. J Burn Care Res. 2013; 34(4):e250-56.

Istre GR, McCoy MA, Osborn L, Barnard JJ, Bolton A. Deaths and injuries from house fires. N Engl J Med. 2001; 344(25):1911-16.

Jackson M, Wilson J, Akoto J, Dixon S, Jacobs DE, Ballesteros MF. Evaluation of fire-safety programs that use 10-year smoke alarms. J Community Health. 2010; 35(5):543-48.

Karter MJ. Fire loss in the United States during 2012. NFPA J. 2013 [cited 2014 Mar 4]. Available from: https://www.nfpa.org/newsandpublications/nfpajournal/2013/september-october-2013/features/fire-loss-in-the-united-statesduring-2012.

Liu Y, Holland AE, Mack K, Diekman S. Disparities in the prevalence of smoke alarms in U.S. households: conclusions drawn from published case studies. J Safety Res. 2011; 42(5):409-13.

McDonald E, Shields W, Perry E, Gielen AC. Applying the health belief model to a home safety intervention in an urban environment. Abstract presented at: American Public Health Association Annual Meeting, 2013; Boston, MA. 
Peek-Asa C, Yang J, Hamann C, Jones MP, Young T, Zwerling C. Smoke alarm and battery function 42 months after installation: a randomized trial. Am J Prev Med. 2010; 39(4):368-71.

Schachterle SE, Bishai D, Shields W, Stepnitz R, Gielen AC. Proximity to vacant buildings is associated with increased fire risk in Baltimore, Maryland, homes. Inj Prev. 2012; 18(2):98-102.

Shai D. Income, housing, and fire injuries: a census tract analysis. Public Health Rep. 2006; 121(2):149-54.

Sidman EA, Grossman DC, Mueller BA. Comprehensive smoke alarm coverage in lower economic status homes: alarm presence, functionality, and placement. J Community Health. 2011; 36:525-33.

Stepnitz R, Shields W, McDonald E, Gielen A. Validity of smoke alarm self-report measures and reasons for over-reporting. Inj Prev. 2012; 18(5):298-302.

Stone KE, Eastman EM, Gielen AC, Squires B, Hicks G, Kaplin D, Serwint JR. Home safety in inner cities: prevalence and feasibility of home safety-product use in inner-city housing. Pediatrics. 2007; 120(2):e345-53.

Ta VM, Frattaroli S, Bergen G, Gielen AC. Evaluated community fire safety interventions in the United States: a review of current literature. J Community Health. 2006; 31(3):176-97.

Yang J, Peek-Asa C, Jones MP, Nordstrom DL, Taylor C, Young TL, Zwerling C. Smoke alarms by type and battery life in rural households: a randomized controlled trial. Am J Prev Med. 2008; 35(1):20-4.

doi:10.1186/s40621-014-0030-3

Cite this article as: Gielen et al: Changes in smoke alarm coverage following two fire department home visiting programs: what predicts success? Injury Epidemiology 2014 1:30.

\section{Submit your manuscript to a SpringerOpen ${ }^{\circ}$ journal and benefit from:}

- Convenient online submission

- Rigorous peer review

- Immediate publication on acceptance

- Open access: articles freely available online

- High visibility within the field

- Retaining the copyright to your article 